\title{
Perfil de autoanticorpos e correlaçáo clínica em um grupo de pacientes com esclerose sistêmica na regiáo sul do Brasil
}

Carolina de Souza Müller ${ }^{1}$, Eduardo dos Santos Paiva², Valderílio Feijó Azevedo ${ }^{3}$, Sebastião Cezar Radominski ${ }^{4}$, José Hermênio Cavalcante Lima Filho ${ }^{5}$

\begin{abstract}
RESUMO
Objetivos: Este estudo visou à análise das manifestações da esclerose sistêmica (ES), com ênfase na pesquisa dos autoanticorpos e de suas correlações clínicas, na população de pacientes em acompanhamento no ambulatório de ES do Hospital de Clínicas da Universidade Federal do Paraná. Metodologia: Realizou-se um estudo transversal com 96 pacientes em acompanhamento no ambulatório de ES do hospital, entre setembro de 2007 e setembro de 2009. Resultados: A maioria dos pacientes era do sexo feminino, com idade entre a quarta e quinta décadas e tempo de doença com mediana de 10 anos. A forma cutânea limitada de ES foi a mais prevalente. Na análise dos autoanticorpos, o anticorpo anticentromérico (ACA) associou-se a ES forma limitada, idade mais avançada ao diagnóstico, maior tempo de doença, intervalo maior entre o surgimento do fenômeno de Raynaud (FRy) e o primeiro sintoma não FRy, maior prevalência de hipertensão arterial sistêmica (HAS) e de bloqueios de condução cardíaca. O anticorpo antitopoisomerase-1 (antitopo-1, previamente denominado anti-Scl-70) foi mais comum na forma difusa da ES, na presença de doença ativa e de úlceras digitais. O anticorpo anti-RNA polimerase III (antipol III) correlacionou-se com a forma difusa de ES, presença de doença ativa e sinovite. Conclusões: Este estudo vem ressaltar e ratificar o papel relevante dos autoanticorpos na avaliação dos pacientes com ES, sendo possível correlacionar o perfil autoimune dessa população com manifestações específicas da doença.
\end{abstract}

Palavras-chave: Escleroderma sistêmico; autoimunidade; autoanticorpos.

[Rev Bras Reumatol 2011;51(4):314-24] CElsevier Editora Ltda

\section{INTRODUÇÃO}

A esclerose sistêmica (ES), também denominada esclerodermia (skleros: duro; derma: pele), é uma doença crônica, de etiologia ainda desconhecida, e que acomete múltiplos sistemas orgânicos. Caracteriza-se por anormalidades estruturais e funcionais de pequenos vasos sanguíneos, fibrose da pele e de órgãos internos e desregulação imune. Os principais subtipos de ES compreendem a forma limitada e difusa, baseando-se na extensão do acometimento cutâneo pela doença. ${ }^{1}$ Tanto na forma difusa quanto limitada da ES, os autoanticorpos estão comumente presentes. Discute-se o papel dos autoanticorpos na patogênese da ES, embora correlações entre os autoanticorpos e manifestações clínicas da doença estejam bem estabelecidas. ${ }^{2}$ $\mathrm{Na}$ ES, o fator antinuclear (FAN) está presente em mais de $95 \%$ dos pacientes, ${ }^{3,4}$ independentemente da forma clínica da doença. $\mathrm{O}$ anticorpo anticentromérico (ACA) tem como alvo proteínas centroméricas do núcleo celular. Está associado à forma limitada

Recebido em 17/01/2011. Aprovado, após revisão, em 30/4/2011. Comitê de Ética: 156084. Os autores declaram a inexistência de conflitos de interesse. Serviço de Reumatologia do Hospital de Clínicas da UFPR, Brasil.

1. Mestre em Medicina Interna; Médica-assistente do ambulatório de esclerose sistêmica do HC- UFPR

2. Professor da Disciplina de Reumatologia, UFPR; Chefe do ambulatório de fibromialgia do HC-UFPR

3. Professor da Disciplina de Reumatologia, UFPR; Chefe do ambulatório de espondiloartrites, gota e esclerose sistêmica do HC-UFPR

4. Professor da Disciplina de Reumatologia, UFPR; Chefe do Serviço de Reumatologia do HC-UFPR

5. Médico do Serviço de Alergologia e Imunologia Clínica, McMaster University, Ontário, Canadá; Pós-doutorado, Massachusetts General Hospital, Harvard Medical School

Correspondência para: Carolina de Souza Müller. Rua Padre Camargo, 549 cjs. 23 e 24. Curitiba, PR. Brasil. CEP 80060-240. E-mail: carolinadesmuller@yahoo.com.br 
da ES e demonstra um papel protetor na doença, uma vez que se associa à menor incidência de fibrose pulmonar. ${ }^{4} \mathrm{O}$ anticorpo antitopoisomerase-1 (antitopo-1), presente em até 20\%-30\% dos pacientes esclerodérmicos, está associado à forma difusa da ES; traduz doença mais grave, de pior prognóstico e de maior mortalidade. ${ }^{4} \mathrm{O}$ anticorpo anti-RNA polimerase III (antipol III) também confere à doença um prognóstico reservado; associa-se à ES forma difusa, crise renal esclerodérmica e maior mortalidade. ${ }^{5} \mathrm{O}$ ambulatório de ES do Hospital de Clínicas da UFPR atende mais de 120 pacientes com a doença. Este estudo visou a identificação, registro e análise dessa população de pacientes, a fim de descrevê-la nos aspectos demográfico, clínico e laboratorial, com ênfase no perfil imunológico.

\section{PACIENTES E MÉTODOS}

Participaram do estudo pacientes com ES em acompanhamento no Serviço de Reumatologia do Hospital de Clínicas das UFPR. O período de inclusão no estudo foi de setembro de 2007 a setembro de 2009. Um termo de consentimento escrito, aprovado pelo Comitê de Ética em Pesquisa em Seres Humanos do hospital foi obtido de todos os pacientes pelo seu médico-examinador, previamente à sua inclusão no estudo. A admissão dos pacientes no estudo respeitou aos seguintes critérios de inclusão: a) acompanhamento no ambulatório de ES do Serviço de Reumatologia do Hospital de Clínicas da UFPR; b) diagnóstico de ES de acordo com os critérios de classificação definidos pelo ACR (American College of Rheumatology); ${ }^{6} \mathrm{c}$ ) idade superior a 16 anos. Foram considerados inelegíveis para o estudo: a) pacientes com doenças scleroderma-like; b) pacientes com esclerodermia localizada; c) pacientes com doença mista do tecido conectivo; d) pacientes que se recusaram a assinar o termo de consentimento informado. Inicialmente, foram avaliados 108 pacientes para a entrada no estudo; deste total, 12 pacientes foram excluídos: um com esclerodermia localizada, dois com o diagnóstico de síndrome da pele rígida (stiff skin syndrome) e nove pacientes que não preenchiam os critérios do ACR para classificação de ES. Os 96 pacientes restantes foram incluídos no estudo. Não houve pacientes que, após a inclusão, foram retirados do estudo.

\section{Coleta de dados}

O levantamento dos dados demográficos, clínicos, de exames complementares e do perfil de autoanticorpos seguiu a rotina de atendimento do ambulatório de ES do Hospital de Clínicas da UFPR. Os exames complementares, excetuando-se a dosagem dos autoanticorpos, foram todos realizados no próprio hospital, conforme os valores referenciais de cada serviço. A análise dos autoanticorpos FAN, ACA, antitopo-1 e antipol III foi realizada no laboratório Metanalysis - Centro de Diagnósticos Médicos, em Porto Alegre, Rio Grande do Sul, e custeada pelo Serviço de Reumatologia do Hospital de Clínicas da UFPR.

\section{Análise dos autoanticorpos}

A análise dos autoanticorpos FAN e ACA, por imunofluorescência indireta (IIF) com células HEp-2 (human larynx epidermoid carcinoma cell line), teve triagem em 1:80. ${ }^{7}$ Para a análise dos autoanticorpos antitopo-1, utilizou-se o kit QUANTA LiteTM Scl-70 do Laboratório INOVA (INOVA Diagnostics, Inc; San Diego, CA, USA). De acordo com o fabricante do kit, a amostra foi classificada como não reagente se $<20$ unidades, fracamente reagente entre 20 e 39 unidades, moderadamente reagente entre 40 e 80 unidades e fortemente reagente (valores elevados) se $>80$ unidades. A dosagem do autoanticorpo antipol III foi realizada por ELISA (Enzyme Linked Immunoassay) para a detecção semiquantitativa de anticorpos IgG antipol III no soro dos pacientes. Os kits utilizados foram provenientes dos laboratórios INOVA e MBL (Medical and Biological Laboratories Co. Ltd., Nagoya, Japão). Pelo kit INOVA, a amostra foi classificada como não reagente se $<20$ unidades, fracamente reagente entre 20 e 39 unidades, moderadamente reagente entre 40 e 80 unidades e fortemente reagente (valores elevados) quando $>80$ unidades. Pelo kit $\mathrm{MBL}$, foram considerados positivos valores $\geq 28 \mathrm{U} / \mathrm{mL}$.

\section{Análise estatística}

A análise estatística foi realizada com auxílio do software de estatística JMP $7.0^{\circledR}$, SAS Institute, Inc., Cary, NC. O nível de significância estatística adotado foi de 5\% ( $\mathrm{P}<0,05 \%)$. Dados com distribuição normal foram apresentados como média \pm desvio-padrão e os não normais como média e os valores de interquartis $25 \%$ e $75 \%$. Para comparações de dois dados com distribuição normal foram usados o teste $\chi^{2}$ ou o teste $t$ de Student; para mais de dois dados normais, utilizou-se o ANOVA. Da mesma forma, para comparação de dados não normais, foram usados o teste de Wilcoxon para duas variáveis e o teste de Kruskal-Wallis para acima de duas variáveis. O valor de " $n$ " (número da amostra) dos dados avaliados só foi especificado quando não correspondia à totalidade dos pacientes.

\section{RESULTADOS}

\section{Dados demográficos}

A idade média dos 96 pacientes foi de 49,27 \pm 12,55 anos. Eram do sexo feminino $88(91,67 \%)$ pacientes. O índice de 
massa corporal (IMC) médio $(n=71)$ foi de $23,69 \pm 3,63$. A etnia dos pacientes $(n=92)$ seguiu a seguinte distribuição: 72 brancos (78,26\%), 4 negros (4,35\%) e 16 pardos (17,39\%). Do total dos pacientes incluídos no estudo, $63(65,62 \%)$ apresentavam ES forma cutânea limitada, 25 (26,04\%) forma cutânea difusa e $8(8,33 \%)$ foram classificados como "outras formas" [sobreposição de ES com artrite reumatoide (AR), lúpus eritematoso sistêmico (LES), polimiosite (PM) ou artrite idiopática juvenil (AIJ)]. O tempo de doença estimado dos pacientes teve mediana de 10 anos (5,25 e 21,00 interquartis).

\section{Dados de autoanticorpos}

Para análise dos autoanticorpos dispunha-se da amostra sorológica de 85 pacientes. O FAN era positivo na maioria dos pacientes $(92,94 \%)$, seguindo-se do antipol III $(41,18 \%)$, antitopo-1 $(31,76 \%)$ e ACA (30,59\%). Excetuando-se o anticorpo

\section{Tabela 1}

Correlação entre os autoanticorpos pesquisados e os dados clínicos dos pacientes (anamnese)

\begin{tabular}{|c|c|c|c|}
\hline Dados de Anamnese & ACA reagente & Antitopo-1 reagente & Antipol III reagente \\
\hline $\mathrm{N}^{*}$ & $26(30,59)$ & $27(31,76)$ & $35(41,18)$ \\
\hline Idade*+ & $54,58 \pm 10,53(P<0,01)$ & $45,78 \pm 12,51$ & $47,2 \pm 12,83$ \\
\hline Idade FRy*+ & $32,04 \pm 16,42$ & $37,70 \pm 13,73$ & $33,8 \pm 13,95$ \\
\hline Idade não FRy*+ & $41,23 \pm 13,87$ & $39,93 \pm 12,55$ & $38,43 \pm 12,36$ \\
\hline Tempo de doença*§ & $23,00(9,75$ e 30,75$)(P<0,01)$ & $7,00(4,00$ e 10,00$)(P<0,01)$ & $10,00(6,00$ e 22,00$)$ \\
\hline Intervalo FRy - não FRy*§ & $3,50(0,75$ e 18,50$)(P<0,01)$ & $1,00(0,00$ e 2,00$)(P<0,01)$ & $2,00(0,00$ e 8,00$)$ \\
\hline Queixas esofágicas ${ }^{*}$ & 61,54 & 66,67 & 65,71 \\
\hline Queixas gástricas & 30,77 & 33,33 & 45,71 \\
\hline Queixas intestinais ${ }^{¥}$ & 26,92 & $14,81(P=0,05)$ & 25,71 \\
\hline Dispneia ${ }^{*}$ & 23,08 & 18,52 & 22,86 \\
\hline Palpitações ${ }^{*}$ & $34,61(P=0,07)$ & 14,81 & 20,00 \\
\hline
\end{tabular}

§ média (interquartis 25\% e 75\%); + (média \pm SD); ¥ (\% positividade); * anos; P: nível de significância estatística na comparação entre ACA, antitopo-1 e antipol III; FRy: fenômeno de Raynaud.

\section{Tabela 2}

Correlação entre os autoanticorpos pesquisados e os dados clínicos dos pacientes (exame clínico)

\begin{tabular}{|c|c|c|c|}
\hline Dados do exame clínico & ACA reagente & Antitopo-1 reagente & Antipol III reagente \\
\hline$N^{*}$ & $26(30,59)$ & $27(31,76)$ & $35(41,18)$ \\
\hline Escore cutâneo de Rodnan ${ }^{\S}$ & $11,50(7,75$ e 20,25$)$ & $15,00(9,00$ e 23,00$)$ & $16,00(8,00$ e 22,00$)$ \\
\hline Forma cutânea difusa ${ }^{*}$ & $4,35(\mathrm{P}<0,01)$ & $52,17(\mathrm{P}=0,03)$ & $56,52(P=0,04)$ \\
\hline "Outras formas" & 14,29 & 42,86 & 42,86 \\
\hline Úlceras digitais ${ }^{*}$ & 42,31 & $70,37(P=0,01)$ & 60,00 \\
\hline Sinovite ${ }^{¥}$ & 11,54 & 7,41 & $20,00(P=0,05)$ \\
\hline Joint contractions $¥$ & 30,77 & 25,93 & 40,00 \\
\hline Contraturas articulares ${ }^{*}$ & 0 & 3,70 & 2,86 \\
\hline $\mathrm{HAS}^{¥}$ & $53,85(\mathrm{P}<0,01)$ & $14,81(P=0,04)$ & 34,29 \\
\hline Crise renal ${ }^{*}$ & 0,00 & 0,00 & 0,00 \\
\hline
\end{tabular}

§média (interquartis 25\% e 75\%); ¥ (\% de positividade); P: nível de significância estatística na comparação entre ACA, anti-topo-1 e anti-pol III; FRy: fenômeno de Raynaud; HAS: hipertensão arterial sistêmica. 
antipol III, com valores baixos a intermediários predominantes, a maioria dos demais autoanticorpos apresentou valores elevados. $\mathrm{Na}$ análise do perfil autoimune dos pacientes, as relações dos diferentes autoanticorpos com os resultados dos exames clínicos e complementares foram avaliadas em busca de associações significativas. Quanto ao ACA, a associação foi positiva com: ES forma limitada, idade mais avançada ao diagnóstico, maior tempo de doença, intervalo maior entre o surgimento do fenômeno de Raynaud (FRy) e o primeiro sintoma não FRy, hipertensão arterial sistêmica (HAS) e bloqueios de condução cardíaca. A associação foi negativa com fibrose pulmonar e distúrbio restritivo à prova de função pulmonar. A presença de antitopo-1 mostrou relação com: ES forma difusa, presença de doença ativa (conforme critérios do Grupo Europeu de Estudo da Esclerodermia ${ }^{8}$ e úlceras digitais. Houve associação negativa com queixas intestinais e HAS. Quanto ao anti-pol III, a associação foi positiva com: ES forma difusa, presença de doença ativa e sinovite. Não foram encontradas associações negativas desse autoanticorpo com os parâmetros avaliados. Nenhum paciente apresentou anticorpos antitopo-1 e ACA concomitantemente positivos. No entanto, dos 35 pacientes antipol III positivos, 11 eram concomitantemente reagentes para ACA e 13 para antitopo-1. Os dados dos autoanticorpos ACA, antitopo-1 e antipol III, com relação aos dados clínicos (anamnese e exame clínico) e de exames complementares estão dispostos, respectivamente, nas Tabelas 1, 2 e 3.

\section{DISCUSSÃO}

Neste estudo, foram identificados, registrados e analisados os dados demográficos, clínicos e laboratoriais, com ênfase no perfil imunológico, de 96 pacientes em acompanhamento no ambulatório de ES do Hospital de Clínicas da UFPR. A população estudada era predominantemente da quarta e quinta décadas de vida, compatível com a faixa etária com maior prevalência de ES. ${ }^{4,9,10}$ Para a estimativa da idade à instalação da ES, considerou-se que o início da doença correspondia à data de surgimento do FRy, conforme descrito no estudo do EUSTAR - Eular Scleroderma Trials and Research. ${ }^{4}$ Há outros trabalhos, no entanto, que consideram que o surgimento da ES corresponde à época de surgimento do primeiro sintoma não FRy. ${ }^{11,12}$ Seja o início da doença correspondente ao surgimento do FRy ou do primeiro sintoma não FRY, neste estudo a idade média estimada ao início da ES não era diferente entre as formas clínicas da doença ou entre os gêneros (dados não demonstrados). Por ser a ES mais prevalente no gênero feminino, o pequeno número de pacientes homens nesse estudo não foi surpreendente. Encontramos uma taxa feminino:masculino de 10,2:1, comparável a de outros trabalhos, como o do Reino Unido de $3: 1^{13}$ e Japão, de $14: 1,{ }^{14}$ sendo mais próxima daquela descrita na Islândia, de 8:1. ${ }^{15}$ Dos pacientes incluídos no estudo, a maioria apresentava ES limitada, descrita como a forma clínica mais comum entre os casos prevalentes de ES., ${ }^{416}$ Para análise dos autoanticorpos dispunha-se da amostra

Tabela 3

Correlação entre os autoanticorpos pesquisados e os dados de exames complementares dos pacientes

\begin{tabular}{llll}
\hline Dados de Exames Complementares & ACA reagente & Antitopo-1 reagente & Antipol III reagente \\
\hline$N^{*}$ & $26(30,59)$ & $27(31,76)$ & $35(41,18)$ \\
\hline Proteínas de fase aguda $^{*}$ & 30,77 & $42,31(n=26)$ & 37,14 \\
\hline Proteinúria $^{*}$ & 3,85 & $7,69(n=26)$ & 5,71 \\
\hline Elevação da creatinofosfoquinase & $4,17(n=24)$ & $4,00(n=25)$ & $21,21(n=33)$ \\
\hline Bloqueios de condução cardíaca & $24,00(n=25)(P=0,05)$ & $4,17(n=24)$ & $9,09(n=33)$ \\
Capacidade de difusão de CO + & $67,09 \pm 23,06$ & $64,92 \pm 20,39$ & $69,62 \pm 24,23$ \\
Redução da fração de ejeção do VE & $8,33(n=24)$ & $4,00(n=25)$ & $12,12(n=33)$ \\
Disfunção diastólica & $37,50(n=24)$ & $28,00(n=25)$ & $33,33(n=33)$ \\
Fibrose Rx tórax & $12,00(n=25)(P<0,01)$ & $44,00(n=25)$ & $29,41(n=34)$ \\
Distúrbio restritivo pulmonar & $16,67(n=24)(P=0,04)$ & $36,00(n=25)$ & $33,33(n=33)$ \\
Hipertensão pulmonar & $33,33(n=24)$ & $20,00(n=25)$ & $30,30(n=33)$ \\
Doença ativa & $19,00(n=23)$ & $50,00(n=24)(P<0,01)$ & $40,00(P=0,05)$ \\
\hline
\end{tabular}

+(média \pm SD); $¥$ (\% de positividade); P: nível de significância estatística na comparação entre ACA, anti-topo-1 e anti-pol III; VE: ventrículo esquerdo. 
sorológica de uma parcela dos pacientes. Nos pacientes analisados, encontramos prevalência do FAN, ACA e antitopo-1 similar àquela descrita na literatura. , $, 3,4,17^{2}$ Foram notáveis, no entanto, os valores elevados desses autoanticorpos e a prevalência excepcionalmente alta de pacientes antipol III positivos (41,18\%). Quando comparada a dados de outros trabalhos, a prevalência do anticorpo antipol III na nossa população é, no mínimo, duas vezes superior. ${ }^{17,18} \mathrm{Em}$ um estudo de 2007 ressaltou a elevada prevalência de antipol III nos pacientes americanos (25\% naquela população) quando comparados a pacientes franceses. ${ }^{19}$ Trabalhos que demonstraram associação dos níveis de autoanticorpos, especialmente do antitopo-1, e manifestações clínicas da ES, reforçam o conceito de que os autoanticorpos não são apenas um epifenômeno na ES, mas que exercem papel na patogênese da doença. ${ }^{20-22}$ No estudo do EUSTAR, o status dos autoanticorpos contribuiu mais para as complicações sistêmicas do que o fez o subtipo clínico de ES. ${ }^{4} \mathrm{Se}$, na literatura, níveis elevados de antitopo-1 mostraram relação com doença em atividade e de maior gravidade, consideramos que os elevados níveis de antitopo-1 e dos demais autoanticorpos desse estudo também reflitam uma população mais gravemente acometida. A avaliação de pacientes provenientes de um centro terciário possivelmente contribuiu para a seleção de casos mais graves. Por se tratar de um estudo transversal, não se fez a análise prospectiva para a confirmação dessa hipótese.

Nesse estudo, como em outros trabalhos, o anticorpo ACA esteve associado a indicadores de melhor prognóstico, diferentemente do anticorpo antitopo-1, associado à ES difusa e de maior gravidade. ${ }^{2,3,23}$ Foi possível aferir um efeito protetor do ACA quanto à fibrose pulmonar e distúrbio restritivo à espirometria; por outro lado, o anticorpo antitopo-1 mostrou associação a maior prevalência de úlceras digitais e doença em atividade, como descrito previamente por outros autores. ${ }^{4}$ Não observamos, no entanto, a correlação do anticorpo ACA e a presença de hipertensão pulmonar (HP), o que seria esperado levando-se em conta a maior prevalência de vasculopatia em pacientes ACA-positivos. ${ }^{3,18}$ Acreditamos que isto se deveu a não termos analisado, devido ao pequeno " $n$ " da amostra, a HP de acordo com a presença, ou não, de fibrose pulmonar à radiografia simples de tórax. Walker et al. ${ }^{4}$ somente encontraram correlação do ACA e HP na ausência de fibrose pulmonar; na presença de fibrose, a correlação foi positiva com antitopo-1.

O anticorpo antipol III, descrito na ES ainda em poucos trabalhos, ${ }^{24-26}$ associou-se também a indicadores de doença mais grave. Na ausência de pacientes com história de crise renal nesse estudo, a associação de antipol III e crise renal esclerodérmica não pôde ser avaliada. Da mesma forma, não foi possível a avaliação de antipol III quanto à mortalidade na ES, por se tratar de um estudo transversal.

Embora estudos demonstrem que os níveis séricos dos autoanticorpos na ES possam correlacionar-se com a atividade de doença, ${ }^{21}$ Reveille e Solomon ${ }^{5}$ consideram que dosagens seriadas dos anticorpos em pacientes com positividade uma vez demonstrada não se mostram justificáveis.

\section{CONCLUSÕES}

O presente estudo possibilitou estabelecer correlações entre o perfil autoimune dos pacientes com ES e manifestações específicas da doença, de forma a ratificar e ressaltar a importância da análise dos autoanticorpos nesta população.

\section{AGRADECIMENTOS}

Ao Dr. Sebastião Cezar Radominski, que, como chefe do Serviço de Reumatologia do Hospital de Clínicas da UFPR, possibilitou a viabilidade de todo o projeto e o fez possível; ao Dr. Carlos Alberto Von Mühlen, que se dedicou pessoalmente ao exame dos autoanticorpos e sempre estimulou a realização desse trabalho.

\section{REFERENCES}

REFERÊNCIAS

1. LeRoy EC, Black C, Fleischmajer R, Jablonska S, Krieg T, Medsger TA, Jr. et al. Scleroderma (systemic sclerosis): classification, subsets and pathogenesis. J Rheumatol 1988; 15(2):202-5.

2. Grassegger A, Pohla-Gubo G, Frauscher M, Hintner H. Autoantibodies in systemic sclerosis (scleroderma): clues for clinical evaluation, prognosis and pathogenesis. Wien Med Wochenschr 2008; 158(12):19-28.

3. Steen VD. Autoantibodies in systemic sclerosis. Semin Arthritis Rheum 2005; 35(1):35-42.

4. Walker UA, Tyndall A, Czirjak L, Denton C, Farge-Bancel D, KowalBielecka $\mathrm{O}$ et al. Clinical risk assessment of organ manifestations in systemic sclerosis: a report from the EULAR Scleroderma Trials And Research group database. Ann Rheum Dis 2007; 66(6):754-63.

5. Reveille JD, Solomon DH. Evidence-based guidelines for the use of immunologic tests: anticentromere, Scl-70, and nucleolar antibodies. Arthritis Rheum 2003; 49(3):399-412.

6. Subcommittee for scleroderma criteria of the American Rheumatism Association. Preliminary criteria for the classification of systemic sclerosis (scleroderma). Diagnostic and Therapeutic Criteria Committee. Arthritis Rheum 1980; 23(5):581-90.

7. Dellavance A, Júnior AG, Nuccitelli B, Taliberti BH, Mühlen CAv, Bichara CDA et al. $3^{\circ}$ Consenso Brasileiro para pesquisa de autoanticorpos em células HEp-2 (FAN). Recomendações para padronização do ensaio de pesquisa de autoanticorpos em células HEp-2, controle de qualidade e associações clínicas. Rev Bras Reumatol 2009; 49(2):89-109. 
8. Valentini G, D’Angelo S, Della Rossa A, Bencivelli W, Bombardieri S. European Scleroderma Study Group to define disease activity criteria for systemic sclerosis. IV. Assessment of skin thickening by modified Rodnan skin score. Ann Rheum Dis 2003; 62(9):904-5.

9. White B. Systemic sclerosis and related syndromes A. Epidemiology, pathology and pathogenesis. In: Klippel JH, editor. Primer on the rheumatic diseases: Arthritis Foundation; 2001. p. 353-57.

10. Steen VD, Medsger TA, Jr. Epidemiology and natural history of systemic sclerosis. Rheum Dis Clin North Am 1990; 16(1):1-10.

11. Lafyatis R, Kissin E, York M, Farina G, Viger K, Fritzler MJ et al. $\mathrm{B}$ cell depletion with rituximab in patients with diffuse cutaneous systemic sclerosis. Arthritis Rheum 2009; 60(2):578-83.

12. Assassi S, Del Junco D, Sutter K, McNearney TA, Reveille JD, Karnavas A et al. Clinical and genetic factors predictive of mortality in early systemic sclerosis. Arthritis Rheum 2009; 61(10):1403-11.

13. Silman A, Jannini S, Symmons D, Bacon P. An epidemiological study of scleroderma in the West Midlands. Br J Rheumatol 1988; 27(4):286-90.

14. Tamaki T, Mori S, Takehara K. Epidemiological study of patients with systemic sclerosis in Tokyo. Arch Dermatol Res 1991; 283(6):366-71.

15. Geirsson AJ, Steinsson K, Guthmundsson S, Sigurthsson V. Systemic sclerosis in Iceland. A nationwide epidemiological study. Ann Rheum Dis 1994; 53(8):502-5.

16. Mayes MD, Lacey JV, Jr., Beebe-Dimmer J, Gillespie BW, Cooper $\mathrm{B}$, Laing TJ et al. Prevalence, incidence, survival, and disease characteristics of systemic sclerosis in a large US population. Arthritis Rheum 2003;4 8(8):2246-55.

17. Ho KT, Reveille JD. The clinical relevance of autoantibodies in scleroderma. Arthritis Res Ther 2003; 5(2):80-93.
18. Walker JG, Fritzler MJ. Update on autoantibodies in systemic sclerosis. Curr Opin Rheumatol 2007; 19(6):580-91.

19. Meyer OC, Fertig N, Lucas M, Somogyi N, Medsger TA, Jr. Disease subsets, antinuclear antibody profile, and clinical features in 127 French and 247 US adult patients with systemic sclerosis. J Rheumatol 2007; 34(1):104-9.

20. Kuwana M, Kaburaki J, Mimori T, Kawakami Y, Tojo T. Longitudinal analysis of autoantibody response to topoisomerase I in systemic sclerosis. Arthritis Rheum. 2000; 43(5):1074-84.

21. Hu PQ, Fertig N, Medsger TA, Jr., Wright TM. Correlation of serum anti-DNA topoisomerase I antibody levels with disease severity and activity in systemic sclerosis. Arthritis Rheum 2003; 48(5):1363-73.

22. Sato S, Hamaguchi Y, Hasegawa M, Takehara K. Clinical significance of anti-topoisomerase I antibody levels determined by ELISA in systemic sclerosis. Rheumatology (Oxford) 2001; 40(10):1135-40.

23. Hesselstrand R, Scheja A, Shen GQ, Wiik A, Akesson A. The association of antinuclear antibodies with organ involvement and survival in systemic sclerosis. Rheumatology (Oxford) 2003; 42(4):534-40.

24. Harvey GR, Butts S, Rands AL, Patel Y, McHugh NJ. Clinical and serological associations with anti-RNA polymerase antibodies in systemic sclerosis. Clin Exp Immunol 1999; 117(2):395-402.

25. Bunn CC, Denton CP, Shi-Wen X, Knight C, Black CM. AntiRNA polymerases and other autoantibody specificities in systemic sclerosis. Br J Rheumatol 1998; 37(1):15-20.

26. Kuwana M, Kaburaki J, Okano Y, Tojo T, Homma M. Clinical and prognostic associations based on serum antinuclear antibodies in Japanese patients with systemic sclerosis. Arthritis Rheum 1994; 37(1):75-83. 